\title{
Development of Event-Type Neutron Imaging Detectors at the Energy-Resolved Neutron Imaging System RADEN at J-PARC
}

\author{
Joseph Don Parker ${ }^{1, a^{*}}$, Masahide Harada ${ }^{2, b}$, Hirotoshi Hayashida ${ }^{1, c}$, \\ Kosuke Hiroi ${ }^{2, \mathrm{~d}}$, Tetsuya $\mathrm{Kai}^{2, \mathrm{e}}$, Yoshihiro Matsumoto ${ }^{1, \mathrm{f}}$, Takeshi Nakatani ${ }^{2, \mathrm{~g}}$, \\ Kenichi Oikawa, ${ }^{2, h}$, Mariko Segawa, ${ }^{2, i}$, Takenao Shinohara ${ }^{2, j}$, Yuhua Su ${ }^{2, k}$, \\ Atsushi Takada ${ }^{3,1}$, Toru Tanimori, ${ }^{3, m}$ and Yoshiaki Kiyanagi, ${ }^{4, n}$ \\ ${ }^{1}$ Neutron Science and Technology Center, CROSS, Tokai, Ibaraki 319-1106 Japan \\ ${ }^{2}$ J-PARC Center, Japan Atomic Energy Agency, Tokai, Ibaraki 319-1195 Japan \\ ${ }^{3}$ Graduate School of Science, Kyoto University, Kyoto 606-8502 Japan \\ ${ }^{4}$ Graduate School of Engineering, Nagoya University, Nagoya, Aichi 464-8603 Japan \\ aj_parker@cross.or.jp, 'barada.masahide@jaea.go.jp, ch_hayashida@cross.or.jp, \\ dkosuke.hiroi@j-parc.jp, ${ }^{\mathrm{d}}$ tetsuya.kai@j-parc.jp, ${ }^{\mathrm{f}} \mathrm{y}$ matsumoto@cross.or.jp, \\ gtakeshi.nakatani@j-parc.jp, 'kenichi.oikawa@j-parc.jp, 'segawa@post.j-parc.jp, \\ jtakenao.shinohara@j-parc.jp, kyuhua.su@j-parc.jp, 'takada@cr.scphys.kyoto-u.ac.jp, \\ mtanimori@cr.scphys.kyoto-u.ac.jp, "kiyanagi@phi.phys.nagoya-u.ac.jp
}

Keywords: Energy-Resolved Neutron Imaging, Neutron Imaging Detectors, Micropattern Detectors, Lithium Glass Scintillators

\begin{abstract}
At the RADEN beam line of the Materials and Life Science Experimental Facility at the Japan Proton Accelerator Research Complex, we combine cutting-edge, event-type imaging detectors with a high-intensity, pulsed neutron beam to perform energy-resolved neutron imaging. In particular, the $\mu$ NID (Micropixel-chamber-based Neutron Imaging Detector), with its unique combination of a large $10 \mathrm{~cm} \times 10 \mathrm{~cm}$ field-of-view, $100 \mu \mathrm{m}$ spatial resolution, and recent improvements in the rate performance to over 1 Mcps, is quickly becoming the main event-type detector for such measurements at RADEN. To improve the ease-of-use of the $\mu$ NID system, we have recently redesigned the control hardware and software to allow full integration into the RADEN experiment control system and developed a web-based user interface for data processing. Further development efforts for the $\mu$ NID, including a new reduced-pitch readout for improved spatial resolution and a boron-converter based $\mu$ NID that achieves a count rate of over 20 Mcps, are also ongoing. In addition, we are studying super resolution methods to improve the spatial resolution of a lithium-glass scintillator pixel detector.
\end{abstract}

\section{Introduction}

At the RADEN instrument [1], located at beam port 22 of the high-intensity, pulsed neutron source of the Materials and Life Science Experimental Facility (MLF) at the Japan Proton Accelerator Research Complex (J-PARC), we take advantage of the accurate measurement of neutron energy by time-of-flight to perform energy-resolved neutron imaging. By analyzing the two-dimensionally resolved, energy-dependent neutron transmission, these techniques can image macroscopic distributions of microscopic properties of bulk materials in situ, including crystallographic structure and residual strain (Bragg-edge transmission [2]), nuclide-specific density and temperature distributions (resonance absorption [3]), and internal/external magnetic fields (polarized neutron imaging [4]). At RADEN, we use advanced, event-type neutron imaging detectors, based on micropattern detectors or fast lithium-glass scintillators with fast, all-digital data acquisition systems for high count rate and sub- $\mu$ s time resolution, to measure the

(c) (1) Content from this work may be used under the terms of the Creative Commons Attribution 3.0 license. Any further distribution of this work must maintain attribution to the author(s) and the title of the work, journal citation and DOI. Published under license by Materials Research Forum LLC. 
energy-dependent neutron transmission at all points over a sample in a single measurement. The quantitative nature and potentially short measurement times make these techniques very attractive for both scientific and industrial applications.

The event-type detectors currently available at RADEN include two micropattern detectors, the $\mu$ NID (Micropixel-chamber-based Neutron Imaging Detector) [5,6] and nGEM (boroncoated Gas Electron Multiplier) [7], and a lithium glass scintillator pixel detector, the LiTA12 ( ${ }^{6} \mathrm{Li}$ Time Analyzer, model 2012) [8]. The performance of these detectors, as measured at RADEN, has recently been presented in Ref. [9], and the results are summarized in Table 1.

Table 1: Performance of event-type detectors at RADEN. 'Peak count-rate capacity' and 'Effective peak count-rate' refer to the global instantaneous peak rates (i.e., peak rates over the entire detector) at the limit of the hardware and with less than $2 \%$ event loss, respectively.

\begin{tabular}{|c|c|c|c|}
\hline Detector & $\mu$ NID & nGEM & LiTA12 \\
\hline Type & Micropattern & Micropattern & Scintillator \\
\hline Neutron converter & ${ }^{3} \mathrm{He}$ & ${ }^{10} \mathrm{~B}$ & ${ }^{6} \mathrm{Li}$ \\
\hline Area $\left[\mathrm{cm}^{2}\right]$ & $10 \times 10$ & $10 \times 10$ & $4.9 \times 4.9$ \\
\hline Time resolution [ns] & 250 & 15 & 40 \\
\hline Spatial resolution [mm] & 0.1 & 1 & 3 \\
\hline Efficiency @25.3 meV [\%] & 26 & 10 & 23 \\
\hline Peak count-rate capacity [Mcps] & 8 & 4.6 & 8 \\
\hline Effective peak count-rate [Mcps] & 1 & 0.18 & 6 \\
\hline
\end{tabular}

We are continually working to improve our event-type neutron imaging detectors for better spatial resolution and shorter measurement times and, as a user facility, to improve the ease-ofuse of their control and analysis software. In particular, we have recently redesigned the $\mu$ NID control software to allow full integration into the experiment control system at RADEN, and we have developed an easy-to-use web-based user interface for data processing. We are also developing a new 215- $\mu \mathrm{m}$ pitch readout for the $\mu$ NID for improved spatial resolution and a $\mu$ NID with boron-based converter for increased count rate via a much-reduced event size. In addition, we have tested super resolution techniques to improve the spatial resolution of the LiTA12 detector. These development efforts are described below.

\section{$\mu$ NID Development}

Integration into the RADEN Control System. RADEN features an experiment device control system based on the IROHA2 software framework developed at the MLF [10]. As its most visible feature, IROHA2 provides a user-friendly web interface, making the system accessible from any device with a web browser. The IROHA2 framework includes device control servers for controlling and monitoring beamline devices, experimental equipment, etc. It also allows the control of detector systems via the DAQ-Middleware framework developed at KEK, which consists of customizable, modular data acquisition software components with a unified interface [11]. Finally, an instrument management server provides overall control of the instrument components and measurement process, and a sequence server provides for automated, multi-step measurements via simple Python-based scripting.

In order to integrate the $\mu$ NID into the RADEN control system, we have developed a new combined DAQ control and power hardware unit, along with the corresponding device control module for IROHA2, and we have rewritten the data acquisition software using the DAQMiddleware framework. (The manufacture of the DAQ control and power unit and coding of the device module and data acquisition software were done by Bee Beans Technologies, Inc.) The 
new DAQ control unit and device control module allow full control of the $\mu$ NID, including detector power, setting of all detector parameters, and real-time monitoring of detector status, all from within the IROHA2 system. Additionally, the IROHA2 system is able to remotely control data acquisition via the new DAQ-Middleware-based data acquisition software, allowing the $\mu$ NID to be used for automated measurements.

As a demonstration of the integrated RADEN/ $\mu$ NID control system, we have carried out a computed tomography measurement as shown in Fig. 1. The sample was an iron step-wedge of size $5 \mathrm{~cm} \times 2.5 \mathrm{~cm} \times 1 \mathrm{~cm}$, and projections from 0 to $180^{\circ}$ in $2^{\circ}$ steps, with an exposure time of 8 minutes per step, were taken with an automated measurement sequence. At the time of the measurement, the MLF beam power was $500 \mathrm{~kW}$, and the observed peak count rate at the detector was 4.4 Mcps. As seen in Fig. 1, we were able to successfully reconstruct a threedimensional image using this data. (The reconstruction and visualization were performed with the commercial software, VGSTUDIO MAX.) In the present case, we have used the full neutron energy spectrum in the reconstruction. Due to the nature of the event-type detectors, however, we can return to the original event data and easily select any neutron energy range for the tomographic reconstruction, adding significant quantitative power and flexibility to computed tomography measurements at RADEN.
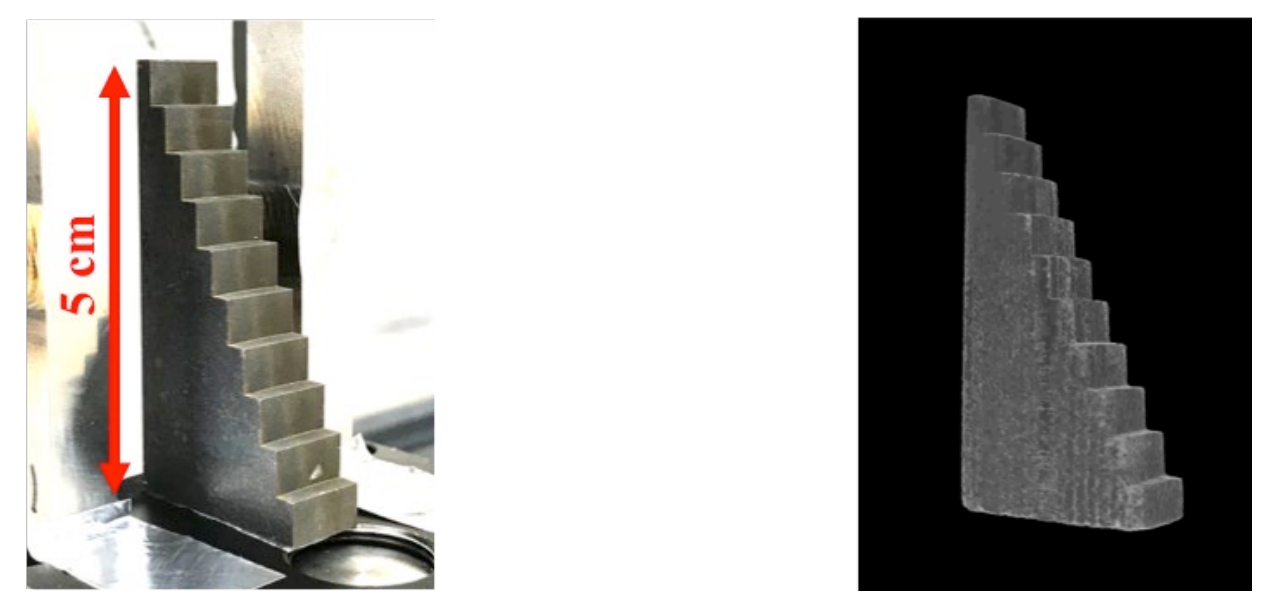

Figure 1. Automated computed tomography measurement with the $\mu$ NID. (Left) Photograph of Fe step-wedge sample. (Right) Three-dimensional image reconstructed from 91 projections (0 to $180^{\circ}, 2^{\circ}$ steps).

Data Processing Software. The $\mu$ NID system produces copious amounts of raw hit data that is currently saved and processed off-line using custom software. This data processing software, which is based on $\mathrm{C}++$ and runs on Linux or macOS, decodes the binary hit data, clusters the hits into neutron events, and calculates the neutron position (using a template fit), time-of-flight, and energy deposition as described in Refs. [5,6]. Over the last several years, we have completely rewritten the data processing software in order to optimize the performance from the ground up, including optimization of the position calculation and implementation of a new clustering algorithm for an improved spatial resolution of $0.1 \mathrm{~mm}$ and an increased rate performance to more than 1 Mcps, respectively, as described in Ref. [9].

To ease the task of data processing, we have also developed a new, web-based graphical user interface (GUI). Modeled loosely after the IROHA2 device control system and written in Python with JavaScript and HTML/CSS, the $\mu$ NID data processing GUI runs as a server-client system, allowing data processing to be performed from any device with a web browser, independent of 
the client operating system. The server-side runs on a Linux or macOS system, where the actual data processing takes place, and acts as the interface between the browser-based GUI and the underlying data processing software. The functionality of the GUI includes user/data management, a guided step-by-step data calibration procedure, data processing job queue, data visualization with interactive plots, and conversion of event data to multipage TIFF. This new GUI system is now in use at RADEN since last year, and we are continuing to make improvements in functionality and performance. The $\mu$ NID data processing software and GUI use only open-source libraries.

New $\mu$ NID Development. We are developing a new readout element with reduced pitch for improved spatial resolution and a $\mu$ NID with a ${ }^{10} \mathrm{~B}$-based neutron converter for increased count rate. These are described in Ref. [9] in detail and are summarized below.

The current readout element of the $\mu$ NID, referred to as the $\mu$ PIC (Micro-Pixel Chamber), is a micropattern readout developed at Kyoto University and manufactured by DaiNippon Printing Company, Ltd. The $\mu$ PIC consists of orthogonal anode and cathode strips with a $400-\mu \mathrm{m}$ pitch on a polyimide substrate and provides gas gain via its unique geometry (maximum gain factor: 6000) [12]. The new readout element is made using MEMS-based (Micro-Electro-Mechanical Systems) manufacturing processes to achieve a reduction in strip pitch from the current $400 \mu \mathrm{m}$ down to $215 \mu \mathrm{m}$, with a corresponding increase in spatial resolution expected after optimization of the gas mixture. Initial testing of a MEMS $\mu$ PIC with a silicon substrate confirmed the basic operation of the new readout. However, instability in the gain arising from the silicon substrate was observed, and we are now investigating an alternate substrate material.

The $\mu$ NID with thin-film boron converter achieves an increase in peak count-rate capacity to over 20 Mcps via a reduced event size as compared to the $\mu$ NID with ${ }^{3} \mathrm{He}$ (i.e., the alpha and $\mathrm{Li}$ nucleus produced by the $\mathrm{n}^{-10} \mathrm{~B}$ reaction travel a much shorter distance in the gas of the detector compared to the proton and triton of the ${ }^{3} \mathrm{He}$ case). A prototype detector has been tested at RADEN, and a 22 Mcps peak count-rate capacity and $0.45 \mathrm{~mm}$ spatial resolution were confirmed, matching expectations. The current boron converter, consisting of a 1.2- $\mu \mathrm{m}$ layer of ${ }^{10} \mathrm{~B}$ deposited on the drift cathode, provides a neutron detection efficiency of only 3 to $5 \%$, and we are now considering ways to increase this detection efficiency.

\section{LiTA12 Development}

The LiTA12 detector, developed at KEK, consists of a $16 \times 16$ array of Li-glass scintillator pixels (type: GS20, size: $2.1 \mathrm{~mm} \times 2.1 \mathrm{~mm} \times 1 \mathrm{~mm}$ ) matched to a Hamamatsu H9500 multianode photomultiplier tube with a 3-mm anode pitch and total area of $4.9 \mathrm{~cm} \times 4.9 \mathrm{~cm}$. Due to the very fast decay time of the Li-glass scintillator, this detector has the potential for very high neutron count rates up to 100 Mcps. The front-end electronics of the current system, however, limit the count rate to 6 Mcps, but this can be increased in the future. This high count-rate capacity and the possibility to easily increase the efficiency by increasing scintillator thickness make the LiTA12 an attractive candidate detector for neutron resonance absorption techniques using epithermal neutrons [13].

The main drawback of the current LiTA12 detector, however, is the poor spatial resolution, which is limited by the 3-mm anode pitch. To address this, we have been investigating so-called super resolution techniques for improved spatial resolution, including charge centroiding and multi-image composition. Charge centroiding is achieved by replacing the scintillator pixels with a single scintillator plate, which allows the light from a single neutron event to spread over multiple anodes. A refined position is then calculated by finding the center-of-gravity of this light distribution. As reported in Ref. [13], tests of such a detector were carried out at BL10 of the J-PARC MLF, and an improvement in spatial resolution to around $0.7 \mathrm{~mm}$ was confirmed. We are also investigating an image compositing technique that uses multiple images taken at 
sub-pixel shifts of the detector to reconstruct an image with improved spatial resolution [14]. Fig. 2 shows the results of a test measurement carried out at RADEN. A total of 36 images of a gadolinium test target [15] were taken by scanning the LiTA12 in 0.5-mm steps in the horizontal and vertical directions. The combined image, constructed very simply by dividing each 3-mm pixel into a $6 \times 6$ grid and placing the pixel values from each image at the corresponding grid point, indicates that the reconstruction of a higher spatial resolution image may be possible from this data. Based on these results, we are now considering a more sophisticated image reconstruction technique.
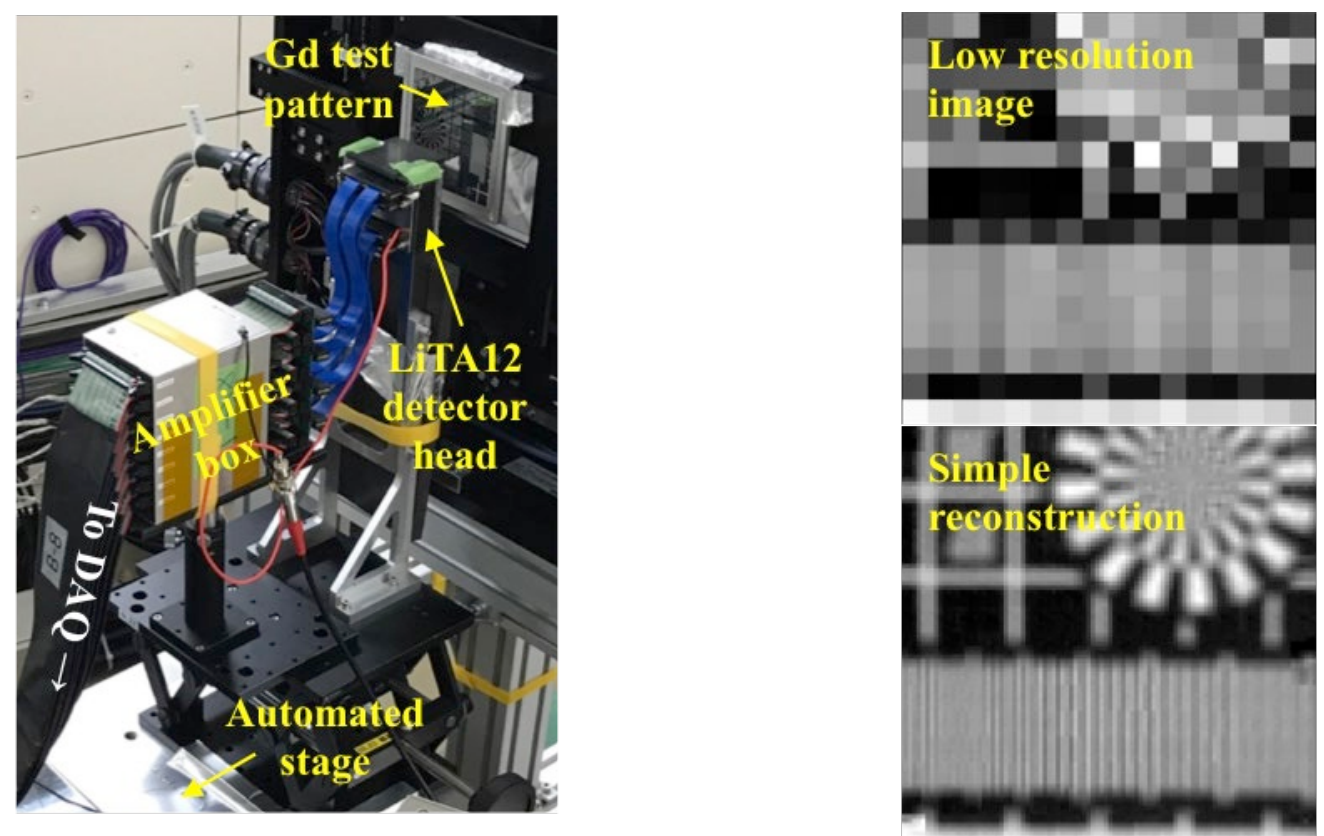

Figure 2. Multi-image compositing with the LiTA12. (Left) Photograph of the LiTA12 mounted on a remote-controlled stage behind the Gd test pattern. (Right, top) Single image taken by the LiTA12 with a $4.9 \mathrm{~cm} \times 4.9 \mathrm{~cm}$ field of view and 3-mm pixel size. (Right, bottom) Composited image combining 36 separate measurements taken at $0.5 \mathrm{~mm}$ steps in the horizontal and vertical directions.

\section{Summary}

At RADEN, we are continuing development of our event-type neutron imaging detectors to better meet the requirements of energy-resolved neutron imaging techniques. For the $\mu$ NID, we have integrated the detector into the RADEN experiment device control system and redesigned the data processing software for an overall improvement in performance and ease-of-use. We are also working to provide improved spatial resolution and increased peak count-rate capacity up to 22 Mcps for the $\mu$ NID by developing a new readout element with reduced pitch and a $\mu$ NID with boron-based converter for reduced event size, respectively. Additionally, we are investigating super resolution techniques to improve the spatial resolution of the LiTA12 detector from the current $3 \mathrm{~mm}$ down to $0.7 \mathrm{~mm}$ or less.

\section{Acknowledgements}

This work was partially supported by the Momose Quantum Beam Phase Imaging Project, ERATO, JST (Grant No. JPMJER1403). Detector testing at RADEN was carried out under Instrument Group Proposal Nos. 2017I0022 and 2018I0022 and CROSS Development Proposal Nos. 2017C0004 and 2018C0002. 


\section{References}

[1] T. Shinohara et al., Final design of the energy-resolved neutron imaging system "RADEN" at J-PARC, J. Phys.: Conf. Series 746 (2016) 012007. https://doi.org/10.1088/17426596/746/1/012007

[2] H. Sato, O. Takada, K. Iwase, T. Kamiyama, and Y. Kiyanagi, Imaging of a spatial distribution of preferred orientation of crystallites by pulsed neutron Bragg edge transmission, J. Phys.: Conf. Series 251 (2010) 012070. https://doi.org/10.1088/1742-6596/251/1/012070

[3] H. Sato, T. Kamiyama, and Y. Kiyanagi, Pulsed neutron imaging using resonance transmission spectroscopy, Nucl. Instr. and Meth. A 605 (2009) 36-39. https://doi.org/10.1016/j.nima.2009.01.124

[4] T. Shinohara et al., Quantitative magnetic field imaging by polarized pulsed neutrons at JPARC, Nucl. Instr. and Meth. A 651 (2011) 121-125. https://doi.org/10.1016/j.nima.2011.01.099

[5] J.D. Parker et al., Neutron imaging detector based on the $\mu$ PIC micro-pixel chamber, Nucl. Instr. and Meth. A 697 (2013) 23-31. https://doi.org/10.1016/j.nima.2012.08.036

[6] J.D. Parker et al., Spatial resolution of a $\mu$ PIC-based neutron imaging detector, Nucl. Instr. and Meth. A 726 (2013) 155-161. https://doi.org/10.1016/j.nima.2013.06.001

[7] S. Uno, T. Uchida, M. Sekimoto, T. Murakami, K. Miyama, M. Shoji, E. Nakano, and T. Koike, Development of a two-dimensional gaseous detector for energy-selective neutron radiography, Phys. Proc. 37 (2012) 600-605. https://doi.org/10.1016/j.phpro.2012.01.035

[8] S. Satoh, Development of a new exclusive function for a 2012 model ${ }^{6} \mathrm{Li}$ time analyzer neutron detector system, JPS Conf. Proc. 8 (2015) 051001. https://doi.org/10.7566/JPSCP.8.051001

[9] J.D. Parker et al., Development of energy-resolved neutron imaging detectors at RADEN, JPS Conf. Proc. 22 (2018) 011022. https://doi.org/10.7566/JPSCP.22.011022

[10] T. Nakatani, Y. Inamura, T. Ito, and T. Otomo, The control software framework of the web base, JPS Conf. Proc. 8 (2015) 036013. https://doi.org/10.7566/JPSCP.8.036013

[11] H. Maeda, Y. Nagasaka, H. Sendai, E. Inoue, E. Hamada, T. Kotoku, N. Ando, S. Ajimura, and M. Wada, Control functionality of DAQ-Middleware, J. Phys.: Conf. Series 513 (2014) 012020. https://doi.org/10.1088/1742-6596/513/1/012020

[12] A. Ochi, T. Nagayoshi, S. Koishi, T. Tanimori, T. Nagae, and M. Nakamura, A new design of the gaseous imaging detector: Micro Pixel Chamber, Nucl. Instr. and Meth. A 471 (2001) 264267. https://doi.org/10.1016/S0168-9002(01)00996-2

[13] T. Kai et al., Characteristics of the 2012 model lithium-6 time-analyzer neutron detector (LiTA12) system as a high efficiency detector for resonance absorption imaging, Phys. B, in press. (DOI:10.1016/j.physb.2017.11.086)

[14] S. Farsiu, M.D. Robinson, M. Elad, and P. Milanfar, Fast and robust multiframe super resolution, IEEE Trans. Image Proc. 13 (2004) 1327-1344.

https://doi.org/10.1109/TIP.2004.834669

[15] M. Segawa et al., Spatial resolution test targets made of gadolinium and gold for conventional and resonance neutron imaging, JPS Conf. Proc. 22 (2018) 011028.

https://doi.org/10.7566/JPSCP.22.011028 